\title{
CONCOMITANT FIBROMYALGIA IN RHEUMATOID ARTHRITIS: CHALLENGES IN DIAGNOSIS AND DISEASE ASSESSMENT
}

\author{
Linda-Jessica Ghib, Laura Muntean, Maria-Magdalena Tamas, Simona Rednic \\ Department of Rheumatology, Iuliu Hatieganu University of Medicine and Pharmacy, Cluj-Napoca
}

\begin{abstract}
Fibromyalgia, characterized by chronic generalized pain and multiple somatic symptoms, is frequently associated with rheumatoid arthritis. This association causes worse disease outcomes and poses challenges in rheumatoid arthritis disease assessment. Recent studies found that fibromyalgia is a disease continuum. Varying degrees of symptoms can be present in patients with rheumatoid arthritis even if they do not satisfy diagnosis criteria for fibromyalgia. Classification criteria for fibromyalgia are recommended for use in research, but in clinical practice diagnosis should be based on physician judgment. Rheumatoid arthritis disease activity scores should be used with caution in patients with concomitant fibromyalgia because they are disproportionately high based on subjective variables, not inflammation. Other means of assessing disease activity such as ultrasonography or inflammation markers can be employed when trying to distinguish between inflammatory and non-inflammatory pain in patients with rheumatoid arthritis and concomitant fibromyalgia.
\end{abstract}

Keywords: pain, polysymptomatic distress, disease activity scores

\section{INTRODUCTION}

Fibromyalgia (FM) is a chronic disease characterized by widespread musculoskeletal pain, fatigue, sleep and cognitive disturbances, with a prevalence of $2 \%$ in the general population (1). Although most of the research performed since the development of the 1990 ACR Classification criteria for fibromyalgia focused on primary FM, in recent years FM associated with other autoimmune or inflammatory conditions has gained much interest. Prevalence of concomitant FM in rheumatoid arthritis (RA) patients is estimated to be $10-20 \%(2-4)$, and is a cause of higher disability and worse disease outcomes (5). Several factors have been linked to the development of FM in RA, including low socio-economic status, psychological distress, comorbidity and pain, but determining factors need further research (6).

A recent study has concluded that FM is not a distinct condition, but the extreme of a disease continuum. As such, patients who do not satisfy diagnostic criteria will have FM features that influence RA prognosis and these symptoms need to be ad- dressed in order to obtain the best therapeutic outcomes (7).

RA treatment is guided by the "treat to target" strategy, which aims to reach and maintain remission or low disease activity as measured by composite disease activity scores (8). These scores have proven to be disproportionately high in patients with fibromyalgic RA (FRA) because of higher subjective measures such as number of tender joints and patients global assessment of disease activity (9). This raised the possibility that patients with FRA would receive aggressive immunosuppressive treatment despite not having active inflammation (9). A recent study by Lage-Hasen et al. (10) concluded that FRA is associated with more frequent use of biological therapy and higher DAS28 score. Because there is no validated biomarker that can measure FM severity or activity, distinguishing between active inflammation and non-inflammatory pain in FRA can pose a real challenge to the clinician. Several methods have been proposed, including ultrasound assessment and multibiomarker activity score (11).

\footnotetext{
Correspondence address:

Linda-Jessica Ghib, MD, Rheumatology Department, 2-4 Clinicilor Street, Cluj-Napoca, Romania

E-mail: ghib.linda@umfcluj.ro
} 
The aim of this paper is to review recent issues concerning diagnosis and disease assessment of FRA. We summarize diagnostic criteria and strategies, possible determining factors for FRA, the concept of polysymptomatic distress (PSD), FM and RA assessment in the context of FRA.

\section{Diagnosis of FM in patients with RA}

Several diagnostic criteria exist for FM, but there is no consensus about which one should be used. Most of these criteria have been developed and validated on FM populations and can be applied in patients with FRA.

The first criteria for FM diagnosis that were endorsed by the ACR were the 1990 ACR classification criteria. A positive diagnosis of FM was made if the patient satisfied two criteria: presence of widespread pain, defined as pain on the right and left side of the body, above and below the waist and also axi-

TABLE 1. The 1990 American College of Rheumatology Criteria for the Classification of fibromyalgia (12)

\begin{tabular}{|c|}
\hline Both of the following criteria have to be satisfied: \\
\hline $\begin{array}{l}\text { 1. History of chronic widespread pain } \\
\text { Pain is considered widespread when all of the following are } \\
\text { present: } \\
\text { - pain in the left side of the body, pain in the right side of } \\
\text { the body, } \\
\text { - pain above the waist and pain below the waist. } \\
\text { - axial skeletal pain (cervical spine or anterior chest or tho- } \\
\text { racic spine or low back) must be present. } \\
\text { Shoulder and buttock pain is considered as pain for each involved } \\
\text { side. 'Low back' pain is considered lower segment pain. }\end{array}$ \\
\hline $\begin{array}{l}\text { 2. Pain in } 11 \text { of } 18 \text { tender point sites on digital palpation } \\
\text { Definition: Pain, on digital palpation, must be present in at } \\
\text { least } 11 \text { of the following } 18 \text { sites: } \\
\text { - Occiput: Bilateral, at the suboccipital muscle insertions. } \\
\text { - Low cervical: bilateral, at the anterior aspects of the inter- } \\
\text { transverse spaces at C5-C7. } \\
\text { - Trapezius: bilateral, at the midpoint of the upper border. } \\
\text { - Supraspinatus: bilateral, at origins, above the scapula } \\
\text { spine near the medial border. } \\
\text { - Second rib: bilateral, at the second costochondral junc- } \\
\text { tions, just lateral to the junctions on upper surfaces. } \\
\text { - Lateral epicondyle: bilateral, } 2 \text { cm distal to the epicondyles. } \\
\text { - Gluteal: bilateral, in upper outer quadrants of buttocks in } \\
\text { anterior fold of muscle. } \\
\text { - Greater trochanter: bilateral, posterior to the trochanteric } \\
\text { prominence. } \\
\text { - Knee: bilateral, at the medial fat pad proximal to the joint } \\
\text { line. } \\
\text { - Digital palpation should be performed with an approxi- } \\
\text { mate force of } 4 \text { kg. } \\
\text { must state that the palpation was painful. 'Tender' is not to } \\
\text { be considered 'painful.' } \\
\text { Widespread pain must have been present for at least } 3 \text { months. } \\
\text { The presence of a second clinical disorder does not exclude } \\
\text { the diagnosis of fibromyalgia. }\end{array}$ \\
\hline
\end{tabular}

al skeletal pain, and tenderness in 11 out of 18 predefined tender points (Table 1). The validation study for these criteria found that they can be applied to both primary FM and FM occurring in the presence of other diseases. Also, it was emphasized that FM is not an exclusionary diagnosis and no further tests are needed if criteria are satisfied (12). One of the most important disadvantages of the 1990 ACR classification criteria is that they were intended for research and are not suitable for clinical practice (13). Also, they omit key symptoms of fibromyalgia such as fatigue, sleep dysfunction and cognitive impairment (14). Many concerns were raised about tender point examination. First of all, they display evaluator variability, they are difficult to perform in daily practice and cannot be used to evaluate the change in disease severity (15). Consequently, Wolfe et al developed the 2010 ACR preliminary diagnostic criteria, which aimed to solve the issues stated above of the ACR 1990 classification criteria. These criteria use a measure of bodily pain, the Widespread Pain Index (WPI) and the Symptom Severity (SS) scale, which includes fatigue, non restorative sleep and cognitive symptoms (Table 2) (14). These criteria have been modified in 2011 into a self reported patient survey, making them more accessible for use in primary care and research (16). Jones et al (17) evaluated the prevalence of FM in the general population comparatively by applying these 3 sets of criteria for FM and found a significantly higher prevalence when the modified 2010 ACR classification criteria were employed. These findings raise the question whether the modified 2010 criteria overestimate FM prevalence. Another finding was that the female/male ratio was much smaller for the 2010 modified criteria than the 1990 and 2010 criteria (17). The lower female/male ration can be explained by the fact that female are more sensitive to pain than men, so, by using the tender point count as a criteria for diagnosis FM prevalence in women might be overestimated (18). It is important to mention that the ACR has not endorsed the 2010 ACR preliminary classification criteria nor the modified 2010 Classification criteria because "endorsement of diagnostic criteria can negatively impact access to care and appropriate treatment for patients" and the diagnosis should be based on physician decision (19).

Benett et al proposed in 2013 a new set of criteria, the Alternative Diagnostic Criteria (2013AltCr), based on a 28 pain location scale and 10 symptom 
TABLE 2. The 2010 ACR preliminary classification criteria for fibromyalgia (14)

\begin{tabular}{l} 
The following three conditions must be met: \\
1. Widespread Pain Index (WPI) $\geq 7$ and Symptom Severity \\
(SS) scale $\geq 5$ or WPI $3-6$ and SS scale score $\geq 9$. \\
2. Symptoms have been present at a similar level for at least \\
$\quad 3$ months. \\
3. The patient does not have a disorder that would otherwise \\
explain the pain. \\
\hline Widespread Pain Index (WPI): \\
Note the number areas in which the patient has had pain over \\
the last week. \\
Score will be between 0 and 19 \\
Shoulder girdle, left; Shoulder girdle, right \\
Hip (buttock, trochanter), left; Hip (buttock, trochanter), right \\
Jaw, left; Jaw, right \\
Upper back; Lower back \\
Upper arm, left; Upper arm, right; Lower arm, left; Lower arm, \\
right \\
Upper leg, left; Upper leg, right; Lower leg, left; Lower leg, right \\
Chest \\
Neck \\
Abdomen \\
SS scale score: Fatigue, waking unrefreshed, cognitive symp- \\
toms \\
For the each of the three symptoms above, indicate the level \\
of severity over the past week using the following scale: \\
$0=$ no problem \\
1 = slight or mild problems, generally mild or intermittent \\
2 = moderate, considerable problems, often present and/or \\
at a moderate level \\
3 = severe: pervasive, continuous, life-disturbing problems \\
Considering somatic symptoms in general, indicate whether \\
the patient has: \\
0 = no symptoms, 1 = few symptoms, 2 = a moderate number \\
of symptoms, 3 = a great deal of symptoms \\
\hline
\end{tabular}

WPI - Widespread Pain Index; SS - Symptom Severity Scale

items from the Symptom Impact Questionnaire. These criteria performed similarly to the 2010 modified ACR preliminary criteria in terms of sensitivity ( $81 \%$ versus $83 \%$ ), but had a higher specificity $(80 \%$ versus $67 \%$ ), and were more efficient in differentiating other chronic pain conditions from FM, but further studies are needed in order to validate these results (20).

Pollard et al (4) developed a diagnostic approach designed specifically for FRA. They used as reference the 1990 ACR classification criteria for FM and showed that FRA diagnosis can be established with a sensitivity of $83 \%$ and a specificity of $80 \%$ by using the difference between tender joint count (TJC) and swollen joint count (SJC) with a cut-off of $\geq 7$.

\section{Determining factors of FM in RA}

The factors that lead to FM development are not well known. Several psychological, social and biological factors have been incriminated for FRA development. Widespread pain in patients who satisfy the 1990 ACR classification criteria is predicted by psychosocial and socioeconomic factors, fatigue, sleep disturbance, stress and behavioral issues (21). Rates and predictors for development of fibromyalgia in a prospective RA cohort were examined by Wolfe et al (6). Fibromyalgia developed at a rate of 5.3 cases per 100 patient years and PSD scores increased towards greater severity of symptoms during the course of the study. Multiple factors contributed to FM development including low socioeconomic status, psychological distress, BMI, PGA, Health Assessment Questionnaire (HAQ) scores, RA severity and PSD score. The most important independent predictors for FRA development were antidepressant use, HAQ, fatigue, WPI and symptom count. Interestingly, during the course of the study patients scores for the PSD were not stable and their FM status changed.

A recent prospective study on the Canadian Early Arthritis cohort examined the incidence and associations between pain, inflammation, psychosocial factors and the presence of FM in an early arthritis cohort (22). The incidence of FM found was between 3.58 and 6.77 cases per 100 person years and was highest in the first year after RA diagnosis. Factors associated with FM diagnosis were pain intensity and poor mental health. One significant finding was that seronegativity for anti CCP was associated with FM. The authors explanation for this result is that physicians might be prone to treat seropositive patients more aggressively or consider pain as inflammatory rather than FM related.

Augmented central pain processing, a key feature of FM, is reflected by lower pain thresholds (23). Disease duration and tender point counts influence pain thresholds in patients with RA, contributing to FRA development (24).

Since antithyroid peroxidase antibodies (TPOAb) are known to be frequent in patients with primary FM (25), Ahmad et al (26) studied the association of TPOAb with FRA in a retrospective study on 204 patients with established RA. They found that FRA diagnosis is associated with the presence of TPOAb. This association was present even after controlling for age, BMI, degenerative disk disease or diabetes and hypothyroidism. There was no influence of CCP antibodies or inflammation markers on the association of TPOAb and FM, suggesting that FM is not influenced by the presence of active inflammation. The authors consider that there is probably no causality between TPOAB and the presence of FM in 
patients with RA, but rather a reflection of immunity dysfunction.

The role of vitamin $\mathrm{D}$ has been explored in pathogenesis of FM (27). Pain is influenced by vitamin D status and plays a significant role in the development and maintenance of chronic pain states (28). The suggested mechanism by which vitamin $D$ is involved in FM relates to the presence of vitamin D receptors and $1 \alpha$ hydroxylase in the hypothalamus and other areas of the central nervous system (29). A recent meta-analysis of 12 studies found that patients with hypovitaminosis D have a higher risk of FM than controls (30). Hypovitaminosis D seems to be correlated with FM severity (31). Gheita et al (32) performed a case control study of vitamin D status in patients with RA and found that patients with FRA had significantly lower levels than patients without FM. These lower levels of vitamin D in patients with FRA compared to patients with RA could be explained by higher disability which, in turn, determines less sun exposure (33). Patients with RA were found to have central system pain amplification expressed by lower pain thresholds and mediated by sleep disturbance.

\section{The concept of polysymptomatic distress}

Several studies suggest that FM is the extreme of a disease continuum. Patients with RA who do not satisfy classification criteria for FM might still have various degrees of FM symptoms, the so called "fibromyalgianes" or polysymptomatic distress. Polysymptomatic distress (PSD) is the sum of the WPI and SS scale with a range between 0 and 31, a measure derived from the 2010 ACR preliminary classification criteria. FM diagnosis is positive if a score of 12 is reached on the PSD scale (34). The idea that fibromyalgia is not a categorical disorder, but an area on a disease continuum was confirmed in a study of Wolfe et al. (35). In this study the authors found that there is a positive correlation between PSD scores and several variables that are typically associated with FM like anxiety, depression and low quality of life. PSD can evaluate FM severity and PSD categories were developed: none: 0-3, mild 4-7, moderate 8-11, severe 12-19, and very severe 20-31. These categories of PSD could replace the FM positive of negative diagnosis and give a more clear idea of FM related symptoms intensity (34).

In a cross-sectional study on 300 RA patients, RA patients were found to exhibit higher mean of PSD
(8.8) scores compared to the general population mean PSD score (3.0). PSD scores greater than the sample mean were associated with worse subjective variables values (TJC, PGA) and determined higher composite RA activity index values. Also, PSD scores predicted all patient reported outcomes. The conclusion was that PSD scores identify disproportionate patient responses irrespective of FM diagnosis. Although the association was weak, PSD correlates with SJC and physician global assessment of disease severity (PhGA). Authors interpreted this as a possible influence of RA activity on an increase of PSD scores (36).

\section{Disease assessment in patients with FRA}

Disease activity in RA is measured by using composite indices such as the disease activity score of 28 joints (DAS28), simplified disease activity index (SDAI), clinical disease activity index (CDAI). These activity measures are used mainly in research and clinical trials, but also in clinical practice in order to guide treatment decisions. The treat to target algorithm proposed by Smolen et al (8) also recommends using disease activity scores in order to monitor treatment response. Reimbursement of biological treatment and monitoring is based on the DAS28 score in some countries (37). A study that compared the DAS28 in patients with RA and patients with FM found that global scores were not statistically different, although individual components such as the TJC, SJC, ESR and PGA were significantly different in between the two groups. SJC and ESR were significantly higher in patients with RA, while TJC and PGA were significantly higher in patients with FM. These results were explained by the presence of pain and decreased pain threshold, not inflammation in patients with FM (38). Several studies $(3,4,39)$ compared DAS28, Health Assessment Questionnaire (HAQ), Short Form Health Survey (SF-36) between patients with FRA and RA. They found that patients with FRA had worse outcomes with regard to DAS28, HAQ and SF-36 compared to RA. The DAS28 score was higher independently of objective evidence of RA activity assessed by SJC and ESR. Also, no significant difference was noted between disease duration, number of DMARDs or rheumatoid factor positivity. A significantly higher proportion of patients with FRA were classified as having high disease activity (DAS28 score $>5.1$ ) compared to RA patients. These results show that DAS28 over- 
estimates disease activity in patients with FRA and could lead to more aggressive treatment in clinical practice. Also, as the majority of clinical trials require high disease activity as inclusion criteria patients with FRA could show worse response in the trial because of persistence of non-inflammatory pain.

Patients with FRA and RA were compared prospectively in an early arthritis cohort of 668 patients concerning disease activity scores. Results were similar to studies on established RA, with FRA patients having higher disease activity scores and HAQ at each evaluation $(6,12,18$ months) compared to those with RA. Treatment caused a similar response in both groups, but scores remained higher in the FRA group and reaching remission or low disease activity was less likely than in the RA group (40).

Thus, DAS28- ESR overestimates disease activity in patients with FRA and using independent variables is recommended rather than disease activity scores in the assessment of such patients. Active disease can be defined as TJC and SJC $\geq 3$ and $\mathrm{ESR} \geq 28 \mathrm{~mm} / \mathrm{h}$ and this definition can better identify active disease in patients with FRA (4).

Tender points and pain threshold seem to influence disease activity scores in patients with RA, irrespective of FM status. Tender point influence on DAS28 score was assessed by Ton et al (41). They examined 200 patients with RA and divided them into 4 groups according to number of tender points: $0,1-6,6-10$ and $\geq 11$ and examined the influence of these groups on DAS28 individual components. With higher numbers of tender points subjective components of DAS28 (TJC, PGA) increased, while SJC and ESR did not. These findings show that the sensitivity of DAS28 to discriminate patients in remission or low disease activity is reduced in the presence of a high number of tender points.

A recent study found that lower pain pressure thresholds at joint (knee) and non-joint (sternum, tibia) sites assessed by using an electronic pressure algometer are associated with higher reported pain, higher subjective DAS28 components and poorer mental health. FM was present in a proportion of $48 \%$ and was associated with lower pain pressure thresholds. Low pain pressure thresholds and FM characteristics such as widespread pain, somatic symptoms, fatigue and low mood were associated. The main cause of increased subjective pain was not ongoing inflammation, as suggested by the lack of significant associations between SJC or ESR with pain pressure thresholds (42).
Although low disease activity and remission have become feasible goals (8), pain persists in a significant proportion of patients (43). FRA patients are less likely to reach remission and low disease activity. Lee et al (44) found that pain, defined as pain of $\geq 4$ on a 0 to 10 scale present in the last week, persists in DAS28 CRP remission in $11.9 \%$ of patients at baseline and $12.5 \%$ at 1 year out of 157 patients included. Variables associated with pain at baseline and at 1 year were PGA, disability, fatigue, sleep disturbance, and self-efficacy. However CRP, SJC, TJC tender-joint count, and Sharp scores were not significantly associated with increased pain severity at baseline or 1 year, suggesting non-inflammatory causes of pain such as fibromyalgia. Another important argument for this theory is that there was a strong association of fibromyalgia features such as fatigue, sleep problems, poor self-efficacy, and baseline and 1-year pain severity. The conclusion of this study was that alternative causes of pain should be addressed when no signs of inflammation are present, but patients still exhibit relevant levels of pain.

A study performed on an observational cohort of RA patients examined the role of depression, anxiety, fatigue and FM on the evaluation of remission status in patients with RA. Patients were divided into 2 groups: those who also fulfilled the ACR/Eular remission criteria (SJC and $\mathrm{TJC} \leq 1, \mathrm{C}$-reactive protein $(\mathrm{CRP}) \leq 1 \mathrm{mg} / \mathrm{dl}$, and $\mathrm{PGA} \leq 1 / 10)$ and those who did not. Out of 87 patients included 32 were in $\mathrm{ACR} /$ Eular remission and 55 were not. A significantly higher number of patients who did not achieve ACR/Eular criteria for remission had depression, anxiety and FM compared to those who were in remission. Although the presence of FM was not significantly higher in the non-ACR/Eular remission group, PSD scores were significantly higher than in the ACR/Eular remission group. (45).

These studies show that there is a need for more objective assessment of disease activity and better tools to discriminate between inflammatory and non-inflammatory causes of pain in RA.

Ultrasonography (US) can be used to detect synovitis, complementary to clinical examination. Synovial hypertrophy and effusions are examined by using gray scale (GS) ultrasound and the neovascularization in the synovial tissue is detected by using the Power Doppler (PD) US (46). Chakr et al (47) performed a case control study of patients with FRA and RA and examined correlations between US 7 joint scores and clinical variables. They found that 
RA patients with FM had higher disease activity scores but similar US scores. GS-US7 correlated with DAS28, SDAI and CDAI in patients with and without FM, while PD-US7 correlated with clinical scores only in patients without FM. We found similar results in our case control study comparing patients with RA, FRA and FM alone in terms of 28 joint ultrasound and clinical measures of disease activity (48). US PD scores can be used to assess active inflammation and US scores could be considered a promising target for disease assessment.

A recent analysis performed on patients enrolled in the United States RA registry found that a significant proportion of patients have clinically active disease despite normal values of ESR and CRP (49). Another study found that the correlation between ESR and CRP is weak, and also that the correlation between ESR, CRP and disease activity in RA is weak (50). Thus, distinguishing active disease based on acute phase reactants like ESR and CRP is not possible for all FRA patients. In recent years a new test has been developed that is more reliable in assessing inflammation than ESR or CRP. It is called the multi-biomarker disease activity (MBDA) and it measures the serum concentrations of 12 biomarkers, including CRP. Its values range between 0-100 (51) and it correlates with DAS28-CRP and other measures of disease activity (52). The MBDA has been shown to predict radiographic progression in both established and early RA $(53,54)$. The utility of the MBDA score in assessing disease activity in patients with FRA has been addressed in a recent study by Lee et al (11). They found that in patients with established RA, MBDA scores were frequently high when CRP was $<1.0 \mathrm{mg} / \mathrm{dl}$. Patients with FRA have similar CRP values and MBDA score, but higher DAS28 CRP. These results show that MBDA is more sensitive in assessing disease activity than CRP when CRP values are low and could better distinguish active inflammation in patients with FRA.
Low MBDA score in a patient with high DAS28 score indicates the presence of non-inflammatory pain.

There is no validated biomarker that can assess disease activity or severity for FM. FM assessment is solely based on patient reporting of severity of symptoms. For this purpose the Fibromyalgia Impact Questionnaire (FIQ) was developed in 1991 (55). Because of issues regarding its difficult scoring system and lack of addressing some relevant symptoms of FM, Bennett et al (56) developed in 2009 a revised version of the FIQ (FIQR). The FIQR consists of 21 items and scores 3 domains: function, overall impact and intensity of symptoms. The total score is 100 , with $30 \%$ of the total score assigned to function, $50 \%$ to symptoms and $20 \%$ to overall impact. Higher scores indicate greater impact of fibromyalgia on functioning (56). Based on a literature search, Wang et al (15) propose a panel of questionnaires that should be used in FM evaluation and monitoring.

\section{CONCLUSION}

This review focuses on recent data regarding diagnosis and disease assessment in FRA. FM diagnosis in patients with RA can be made by using the different ACR criteria or the 2013AltCr since there is no accepted gold standard. Recommendations are that in clinical practice diagnosis should be made based on clinician opinion. It is important to remember that FM is the extreme of a disease continuum and that varying degrees of PSD may influence RA outcomes. RA assessment in the presence of FM should avoid using composite disease activity indexes and focus on individual variables. Additional assessment tools such as US or MBDA can be used when inflammatory and non-inflammatory causes of pain in RA are assessed. Various questionnaires are available for use in order to determine FM severity and evaluate treatment response.

\section{REFERENCES}

1. Queiroz L.P. Worldwide epidemiology of fibromyalgia topical collection on fibromyalgia. Curr Pain Headache Rep. 2013;17(8): 356-62.

2. Wolfe F., Michaud K. Severe rheumatoid arthritis, worse outcomes, comorbid illness, and sociodemographic disadvantage characterize ra patients with fibromyalgia. J Rheumatol. 2004; 31(4):695-700.

3. Ranzolin A., Brenol J.C.T., Bredemeier M., et al. Association of concomitant fibromyalgia with worse disease activity score in 28 joints, health assessment questionnaire, and short form 36

scores in patients with rheumatoid arthritis. Arthritis Rheum. 2009; 61(6):794-800.

4. Pollard L.C., Kingsley G.H., Choy E.H., et al. Fibromyalgic rheumatoid arthritis and disease assessment. Rheumatology. 2010; 49(5):924-8.

5. Wolfe F., Michaud K. Severe Rheumatoid Arthritis, Worse Outcomes, Comorbid IIIness, and Sociodemographic Disadvantage Characterize RA Patients with Fibromyalgia. J Rheumatol. 2004; 31(4):695-700. 
6. Wolfe F., Häuser W., Hassett A.L., et al. The development of fibromyalgia--I: examination of rates and predictors in patients with rheumatoid arthritis (RA). Pain. 2011 Feb;152(2):291-9.

7. Wolfe F., Michaud K., Busch R.E., et al. Polysymptomatic distress in patients with rheumatoid arthritis: understanding disproportionate response and its spectrum. Arthritis Care Res (Hoboken). 2014; 66(10):1465-71

8. Smolen J.S., Aletaha D., Bijlsma J.W.J., et al. Treating rheumatoid arthritis to target: recommendations of an international task force. Ann Rheum Dis. 2010; 69(4):631-7.

9. Ranzolin A., Brenol J.C.T., Bredemeier M., et al. Association of concomitant fibromyalgia with worse disease activity score in 28 joints, health assessment questionnaire, and short form 36 scores in patients with rheumatoid arthritis. Arthritis Care Res. 2009; 61(6):794-800.

10. Lage-Hansen P., Chrysidis S., Lage-Hansen M., et al. Concomitant fibromyalgia in rheumatoid arthritis is associated with the more frequent use of biological therapy: a cross-sectional study. Scand J Rheumatol. 2016; 45(1):45-8.

11. Lee Y.C., Hackett J., Frits M., et al. Multibiomarker disease activity score and $\mathrm{C}$-reactive protein in a cross-sectional observational study of patients with rheumatoid arthritis with and without concomitant fibromyalgia. Rheumatology (Oxford). 2016; 55(4):640-8

12. Wolfe F., Smythe H.A., Yunus M.B., et al. The American College of Rheumatology 1990. Criteria for the classification of fibromyalgia. Report of the Multicenter Criteria Committee. Arthritis Rheum. 1990; 33(2):160-72

13. Wolfe F. Stop using the American College of Rheumatology criteria in the clinic. J Rheumatol. 2003; 30(8):1671-2.

14. Wolfe F., Clauw D.J., Fitzcharles M.A., et al. The American College of Rheumatology preliminary diagnostic criteria for fibromyalgia and measurement of symptom severity. Arthritis Care Res (Hoboken). 2010;62(5):600-10.

15. Wang S-M, Han C, Lee S-J, et al. Fibromyalgia diagnosis: a review of the past, present and future. Expert Rev Neurother. 2015; 15(6):667-79.

16. Wolfe F., Clauw Dj, Fitzcharles M-A, et al. Fibromyalgia Criteria and Severity Scales for Clinical and Epidemiological Studies: A Modification of the ACR Preliminary Diagnostic Criteria for Fibromyalgia. J Rheumatol. 2011; 38(6):1113-22.

17. Jones G.T., Atzeni F., Beasley M., et al. The Prevalence of Fibromyalgia in the General Population: A Comparison of the American College of Rheumatology 1990, 2010, and Modified 2010 Classification Criteria. Arthritis Rheumatol. 2015; 67(2):568-75.

18. Vincent A., Lahr B.D., Wolfe F., et al. Prevalence of Fibromyalgia: A Population-Based Study in Olmsted County, Minnesota, Utilizing the Rochester Epidemiology Project. Mayo Clin. Arthritis Care Res (Hoboken). 2013 May ;65(5):786-92

19. ACR Response to Inquiry on the Fibromyalgia Diagnostic Criteria [Internet]. Available from: http://www.prohealth.com/library/ showarticle.cfm?libid=19470 Accesed on September 5

20. Bennett R.M., Friend R., Marcus D., et al. Criteria for the Diagnosis of Fibromyalgia: Validation of the Modified 2010 Preliminary American College of Rheumatology Criteria and the Development of Alternative Criteria. Arthritis Care Res (Hoboken) 2014; 66(9):1364-73.

21. Mcbeth J., Macfarlane G.J., Hunt I.M., et al. Risk factors for persistent chronic widespread pain: a community-based study. Rheumatology (Oxford). 2001 Jan; 40(1):95-101

22. Lee Y.C., Lu B., Boire G., et al. Fibromyalgia in an Early Arthritis Cohort. Ann Rheum Dis. 2013 Jun; 72(6):949-54.

23. Lee Y.C., Nassikas N.J., Clauw D.J. The role of the central nervous system in the generation and maintenance of chronic pain in rheumatoid arthritis, osteoarthritis and fibromyalgia. Arthritis Res Ther.; 2011; 13(2):211

24. Pollard L.C., Ibrahim F., Choy E.H., Scott D.L. Pain thresholds in rheumatoid arthritis: The effect of tender point counts and disease duration. J Rheumatol. 2012; 39(1):28-31.

25. Ahmad J., Tagoe C.E. Fibromyalgia and chronic widespread pain in autoimmune thyroid disease. Clin Rheumatol. 2014; 33(7):885-91.

26. Ahmad J., Blumen H., Tagoe C.E. Association of antithyroid peroxidase antibody with fibromyalgia in rheumatoid arthritis. Rheumatol Int. 2015; 35(8):1415-21.
27. Karras S., Rapti E., Matsoukas S., et al. Vitamin D in Fibromyalgia: A Causative or Confounding Biological Interplay? Nutrients. 2016; 8(6):343-357

28. Shipton E.A., Shipton E.E. Vitamin D and Pain: Vitamin D and Its Role in the Aetiology and Maintenance of Chronic Pain States and Associated Comorbidities. Pain Res Treat. 2015; 2015: 904967.

29. Eyles D.W., Smith S., Kinobe R., et al. Distribution of the Vitamin $\mathrm{D}$ receptor and $1 \alpha$-hydroxylase in human brain. $\mathrm{J}$ Chem Neuroanat. 2005; 29(1):21-30.

30. Hsiao M-Y, Hung C-Y, Chang K-V, et al. Is Serum Hypovitaminosis D Associated with Chronic Widespread Pain Including Fibromyalgia? A Meta-analysis of Observational Studies Pain Physician. 2015; 18:877-88

31. Armstrong D.J, Meenagh G.K., Bickle l., et al. Vitamin $D$ deficiency is associated with anxiety and depression in fibromyalgia. Clin Rheumatol. 2007; 26(4):551-4.

32. Gheita T.A., Sayed S., Gheita H.A., et al. Vitamin D status in rheumatoid arthritis patients : relation to clinical manifestations, disease activity, quality of life and fibromyalgia syndrome. Int $\mathrm{J}$ Rheum Dis. 2016; 19(3):294-9;

33. Olama S.M., Senna M.K., Elarman M.M., et al. Serum vitamin D level and bone mineral density in premenopausal Egyptian women with fibromyalgia. Rheumatol Int.; 2013 Jan 4; 33(1):185-92.

34. Wolfe F., Walitt B.T., Rasker J.J., et al. The Use of Polysymptomatic Distress Categories in the Evaluation of Fibromyalgia (FM) and FM Severity. J Rheumatol. 2015; 42(8):1494-501

35. Wolfe F., Brähler E., Hinz A., et al. Fibromyalgia prevalence, somatic symptom reporting, and the dimensionality of polysymptomatic distress: Results from a survey of the general population. Arthritis Care Res. 2013; 65(5):777-85.

36. Wolfe F., Michaud K., Busch R.E., et al. Polysymptomatic distress in patients with rheumatoid arthritis: understanding disproportionate response and its spectrum. Arthritis Care Res (Hoboken). 2014; 66(10):1465-71.

37. Orlewska E., Ancuta I., Anic B., et al. Access to biologic treatment for rheumatoid arthritis in Central and Eastern European (CEE) countries. Med Sci Monit. 2011; 17(4):SR1-R13.

38. Leeb B.F., Andel I., Sautner J., et al. The DAS28 in rheumatoid arthritis and fibromyalgia patients. Rheumatology. 2004; 43(1):1504-7.

39. Andersson M.L.E., Svensson B., Bergman S. Chronic widespread pain in patients with rheumatoid arthritis and the relation between pain and disease activity measures over the first 5 years. J Rheumatol. 2013; 40(12):1977-85.

40. Duran J., Combe B., Niu J., et al. The effect on treatment response of fibromyalgic symptoms in early rheumatoid arthritis patients: results from the ESPOIR cohort. Rheumatology (Oxford). 2015; 54(12):2166-70.

41. Ton E., Bakker M.F., Verstappen S.M.M., et al. Look beyond the disease activity score of 28 joints (DAS28): tender points influence the DAS28 in patients with rheumatoid arthritis. J Rheumatol. 2012; 39(1):22-7.

42. Joharatnam N., McWilliams D.F., Wilson D., et al. A crosssectional study of pain sensitivity, disease-activity assessment, mental health, and fibromyalgia status in rheumatoid arthritis. Arthritis Res Ther. 2015 Jan; 17(1):11.

43. Taylor P., Manger B., Alvaro-Gracia J., Johnstone R., GomezReino J., Eberhardt E., et al. Patient perceptions concerning pain management in the treatment of rheumatoid arthritis. J Int Med Res. 2010; 38(4):1213-24.

44. Lee Y.C., Cui J., Lu B., et al. Pain persists in DAS28 rheumatoid arthritis remission but not in ACR/EULAR remission: a longitudinal observational study. Arthritis Res Ther. 2011; 13:R83.

45. Inanc N., Yilmaz-Oner S., Can M., et al. The role of depression, anxiety, fatigue, and fibromyalgia on the evaluation of the remission status in patients with rheumatoid arthritis. J Rheumatol. 2014; 41(9):1755-60.

46. Naredo E., Bonilla G., Gamero F., et al. Assessment of inflammatory activity in rheumatoid arthritis: a comparative study of clinical evaluation with grey scale and power Doppler ultrasonography. Ann Rheum Dis. 2005; 64(3):375-81.

47. da Silva Chakr R.M., Brenol J.C.T., Behar M., et al. Is ultrasound a better target than clinical disease activity scores in rheumatoid 
arthritis with fibromyalgia? A case-control study. PLoS One. 2015 Jan; 10(3):e0118620.

48. Ghib L.J., Tamas M-M, Damian L.O., Felea I., Muntean L.M., Rednic N., et al. The role of ultrasonography in assessing disease activity in patients with rheumatoid arthritis and associated fibromyalgia. Med Ultrason. 2015; 17(3):339-44.

49. Kay J., Morgacheva O., Messing S.P., et al. Clinical disease activity and acute phase reactant levels are discordant among patients with active rheumatoid arthritis: acute phase reactant levels contribute separately to predicting outcome at one year. Arthritis Res Ther. 2014; 16(1):R40.

50. Keenan R.T., Swearingen C.J., Yazici Y. Erythrocyte sedimentation rate and C-reactive protein levels are poorly correlated with clinical measures of disease activity in rheumatoid arthritis, systemic lupus erythematosus and osteoarthritis patients. Clin Exp Rheumatol. 2008; 26(5):814-9.

51. Centola M., Cavet G., Shen Y., et al. Development of a MultiBiomarker Disease Activity Test for Rheumatoid Arthritis. PLoS One. 2013;8(4):e60635
52. Curtis J.R., Van Der Helm-Van Mil A.H., Knevel R., Huizinga T.W., Haney D.J., Shen Y., et al. Validation of a novel multibiomarker test to assess rheumatoid arthritis disease activity. Arthritis Care Res. 2012; 64(12):1794-803.

53. van der Helm-van Mil A.H.M., Knevel R., Cavet G., Huizinga T.W.J., Haney D.J. An evaluation of molecular and clinical remission in rheumatoid arthritis by assessing radiographic progression. Rheumatol. 2013; 52(5):839-46.

54. Hambardzumyan K., Bolce R., Saevarsdottir S., Cruickshank S.E., Sasso E.H., Chernoff D., et al. Pretreatment multi-biomarker disease activity score and radiographic progression in early RA: results from the SWEFOT trial. Ann Rheum Dis. 2015; 74(6):1102-9.

55. Burckhardt C.S., Clark S.R., Bennett R.M. The fibromyalgia impact questionnaire: development and validation. J Rheumatol. 1991; 18(5):728-33.

56. Bennett R.M., Friend R., Jones K.D., Ward R., Han B.K., Ross R.L. The Revised Fibromyalgia Impact Questionnaire (FIQR): validation and psychometric properties. Arthritis Res Ther. 2009; 11 